\title{
Tecnura
}

\section{Determinación de polos de generación distribuida a partir de biomasa residual agrícola en la región Madre de Dios, Perú}

\author{
Determination of distributed generation poles \\ from agricultural residual biomass in Madre de Dios, Peru
}

\author{
Andrés de Jesús Vargas Soplín¹, Judith María Ramírez Candia²
}

Fecha de recepción: 10 de febrero de 2017

Fecha de aceptación: 28 de abril de 2017

Cómo citar: Vargas, A. y Ramírez, J. (2017). Determinación de polos de generación distribuida a partir de biomasa residual agrícola en la región Madre de Dios, Perú. Revista Tecnura, 21(53), 61-77, doi: 10.14483/22487638.11722

\section{Resumen}

Contexto: la región Madre de Dios, Perú, se encuentra condicionada por la forma del asentamiento disperso de sus poblaciones. Esta configuración limita el acceso de la energía a centros poblados rurales, que se encuentra en $69 \%$ en la población rural; por ello, esta investigación tuvo como objetivo principal la determinación de polos de generación distribuida a partir de biomasa residual agrícola en la región de Madre de Dios.

Método: mediante un esquema metodológico de cuatro fases, se analizó la cobertura eléctrica de la región Madre de Dios y se priorizaron centros poblados rurales con mayor posibilidad de energización; posteriormente, se determinó la cantidad de biomasa residual agrícola de los cultivos mayoritarios en la región y su localización geográfica. Seguidamente, se analizaron las opciones tecnológicas de aprovechamiento energético disponibles; por último, se estimó la cantidad de energía aprovechable de la biomasa residual agrícola.

Resultados: se determinaron cinco centros poblados rurales priorizados en Madre de Dios para la energización. En cuanto a la biomasa residual agrícola, ésta se estimó en 6645.08 ton/año para arroz, 5191.20 ton/año para maíz amarillo duro y 7862.37 ton/año para plátano, los tres cultivos mayoritarios en la región. Se estimó que, para el aprovechamiento de cascarilla de arroz, el rango de generación de energía varía de 838.86 a $1319.23 \mathrm{KWh} /$ año mediante gasificadores de lecho móvil, y de 317.99 a 897.56 KWh/año para gasificadores de lecho fluidizado, con lo cual se cubriría la demanda energética de los centros poblados rurales priorizados, que asciende a $211.9 \mathrm{MWh} / \mathrm{año}$.

Conclusiones: la región de Madre de Dios cuenta con potencial de generación de energía eléctrica distribuida que puede cubrir la demanda de los cinco centros poblados priorizados al $100 \%$. Asimismo, la energía generada en la región Madre de Dios mediante lecho fluidizado podría atender la demanda de 1495 habitantes aproximadamente, mientras que mediante lecho móvil se cubriría la demanda de 3945 habitantes aproximadamente.

Palabras clave: biomasa, desarrollo rural, electrificación rural, generación distribuida, Madre de Dios, Perú.

1 Ingeniero ambiental de la Universidad Nacional Agraria La Molina. Lima, Perú. Contacto: 20070133@lamolina.edu.pe

2 Ingeniera en recursos naturales y de energías renovables, magíster en Ciencias Ambientales. Docente de la Universidad Nacional Agraria La Molina. Lima, Perú. Contacto: jramirez@lamolina.edu.pe 


\section{Abstract}

Context: The region Madre de Dios is located in Peru and is conditioned by the dispersed way in which the people establish settlements. This configuration limits the access these rural settlements have to energy (currently, the rural electrification only reaches $31 \%$ ). Thus, this investigation focuses on determining distributed energy poles through the use of residual biomass in Madre de Dios region.

Method: Through a methodological scheme of four phases, the electric coverage in Madre de Dios was analyzed and the rural settlements with better energization chances were prioritized. Next, the amount of agricultural biomass waste of major crops in Madre de Dios was determined and geographically located; then, the technological options of energetic harnessing were analyzed. Finally, the amount of agricultural biomass waste that can be used for energy was estimated.

Results: Five rural settlements were prioritized in Madre de Dios for the possible process of providing energy. The agricultural biomass waste was estimated in 6,645.08 ton/year for rice; 5,191.20 ton/year for yellow corn; and 7,862.37 ton/year for banana. These are the three major crops in the region. For rice husk located in productive areas, the energy was estimated to vary between $838.86 \mathrm{KWh} /$ year and 1,319.23 $\mathrm{KWh} /$ year through the use of mobile bed gasifiers; and it varies from $317.99 \mathrm{KWh} /$ year to 897.56 KWh/year for fluidized bed gasifiers, which meets the energetic demand of the prioritized rural settlements (211.9 MWh/year).

Conclusions: The region of Madre de Dios has potential for generating distributed energy, which could meets the demand of five prioritized rural settlements up to $100 \%$. Likewise, using fluidized bed gasifiers, the energy generated in Madre de Dios could meet the demand of 1495 inhabitants, approximately; and by using of mobile bed gasifiers, the coverage could amount to 3945 inhabitants

Keywords: biomass, distributed generation, Madre de Dios, Peru, rural develpment, rural electricity.

\section{INTRODUCCIÓN}

Sin energía abundante y barata, la base productiva y el tejido social simplemente se desmoronarían; con energía abundante cualquier avance es posible, porque a la larga la producción y el consumo son funciones energéticas (Casilda, 2002). Se puede decir entonces que existe una estrecha relación entre la energía y el desarrollo.

El $88,8 \%$ del territorio peruano tiene cobertura eléctrica y un $63 \%$ en zonas rurales; dentro de la Amazonía peruana, el $32 \%$ de la población de Madre de Dios no cuenta con cobertura eléctrica, encontrándose localizado el $69 \%$ de este grupo en zonas rurales (INEI, 2007). Esto se debe a que la mayor barrera para la ramificación del Sistema Nacional Interconectado (SEIN) es la densa vegetación propia de la región, y la limitada red de vías de comunicación; por esta razón, resulta importante buscar alternativas tecnológicas para provisionar energía en las zonas carentes, a partir de la generación con recursos locales.

Madre de Dios cuenta con el 15,6\% de su territorio para uso agropecuario (IIAP, 2001), actividad que genera residuos aprovechables para la generación de energía, los cuales se denominan como biomasa residual agrícola (Nogués, et al., 2010). La generación de energía por medio de biomasa residual agrícola se puede dar a través de la generación distribuida, consiste en redes de energía in situ para abastecer una demanda local, la cual puede operar tanto de manera aislada como conectada a la red abastecimiento local (Velásquez, et al., 2010).

Sobre la base a los antecedentes previos, esta investigación tuvo como objetivo principal la determinación de polos de generación distribuida a partir de biomasa residual agrícola en la región de Madre de Dios. 


\section{La biomasa residual agrícola}

La biomasa puede ser definida de manera general como las sustancias orgánicas que tienen origen en los compuestos de carbono, formado en la fotosíntesis; desde el punto de vista energético, la biomasa engloba una serie de recursos muy diversos, y llega a ser delimitada y clasificada teniendo en cuenta el binomio recurso-tecnología. La biomasa puede clasificarse como biomasa sólida y líquida, siendo la primera de interés para este presente estudio; a su vez, la biomasa sólida se clasifica en biomasa primaria y secundaria: la primera comprende los cultivos y biomasa forestal que apuntan a fines de producción energética, la segunda incluye los restos agrícolas, forestales o de procesos industriales cuyo fin es distinto a la producción energética (Nogués, et al., 2010).

Dentro de las ventajas de utilizar la biomasa residual con fines energéticos destaca, principalmente, la reducción de la contaminación, la reducción del riesgo de incendios, y el bajo costo de producción, ya que normalmente el dinero que se invierte en producir biomasa corresponde fundamentalmente al costo de la producción de los cultivos, dado que la biomasa residual es un subproducto del proceso productivo agrícola (De Juana, et al., 2007).

Velásquez (2006) sostiene una forma de predicción de la generación potencial de biomasa en campo basándose en las ecuaciones (1) y (2).

$$
\begin{aligned}
& P B_{j}=V_{j} \times \lambda_{j} \\
& P B_{j}=S_{j} \times \delta_{j}
\end{aligned}
$$

En donde:

$P B_{j^{\prime}}$ es la biomasa potencial obtenida en un sistema agrícola de características " $j$ " en toneladas (ton).

$V_{j^{\prime}}$ es la cantidad de recurso obtenido en un sistema productivo de características " $j$ " en una determinada operación. Ejemplo: ton de frutos.

$\lambda_{j^{\prime}}$ es el coeficiente de potencialidad gravimétrico de producción de biomasa en un sistema de características " $j$ ".
$S_{j^{\prime}}$ es la superficie del sistema en características " $j$ ".

$\delta_{j^{\prime}}$ es el coeficiente de potencialidad superficial de producción de biomasa en un sistema de características " $j$ " (ton biomasa residual seca / ha.).

Las variables " $V_{j}^{\prime \prime}$ y " $S_{j}^{\prime \prime}$ se pueden determinar mediante información estadística oficial del organismo estatal competente en cuanto a producción agropecuaria, que en Perú corresponde al Ministerio de Agricultura de la República del Perú (MINAG).

Los coeficientes " $\lambda_{j}$ " $\mathrm{y}$ " $\delta_{j}$ " se pueden determinar en base a revisiones bibliográficas o mediante levantamiento de información in situ.

La biomasa residual agrícola se aprovecha energéticamente mediante la conversión termoquímica, a través de tres mecanismos de conversión: combustión, gasificación y pirólisis. Para la vertiente de la energía distribuida solo se considera la gasificación, ya que es el proceso que mejor se adecua a la misma (Nogués, et al., 2010).

En el proceso de gasificación, la biomasa es transformada en una mezcla de productos gaseosos, líquidos y sólidos mediante su oxidación parcial a altas temperaturas en déficit de oxígeno; esto se realiza para la obtención de un gas que es llamado gas de síntesis o syngas, conformado principalmente por monóxido y dióxido de carbono, hidrógeno, metano y otros hidrocarburos. Su poder calorífico depende de las condiciones del proceso (agente gasificante, temperatura, presión, etc.). Posteriormente, el syngas puede ser aprovechado por motores a gas, turbinas a gas o calderas; la figura 1 muestra el flujo esquemático del proceso de gasificación de la biomasa.

Dentro las tecnologías de gasificación destacan principalmente dos tipos de familias: los gasificadores de lecho fluidizado y los de lecho móvil (contracorriente updraft y equicorriente down$d r a f t)$. El proceso de gasificación posee una serie de etapas en común en ambas familias tecnológicas y otras que son propias de cada una.

Tal como se aprecia en la figura 2, el gasificador de lecho fluidizado es aquel donde la partícula 


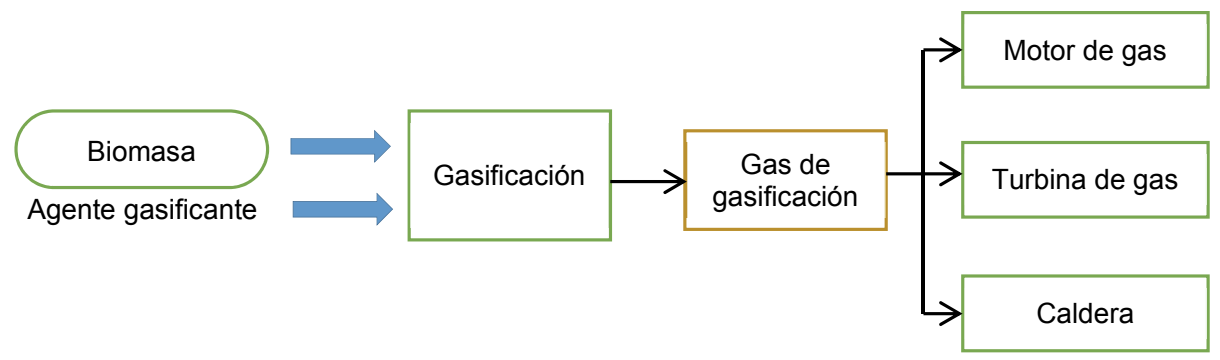

Figura 1. Flujo del proceso de gasificación de la biomasa

Fuente: (Nogués, et al., 2010)

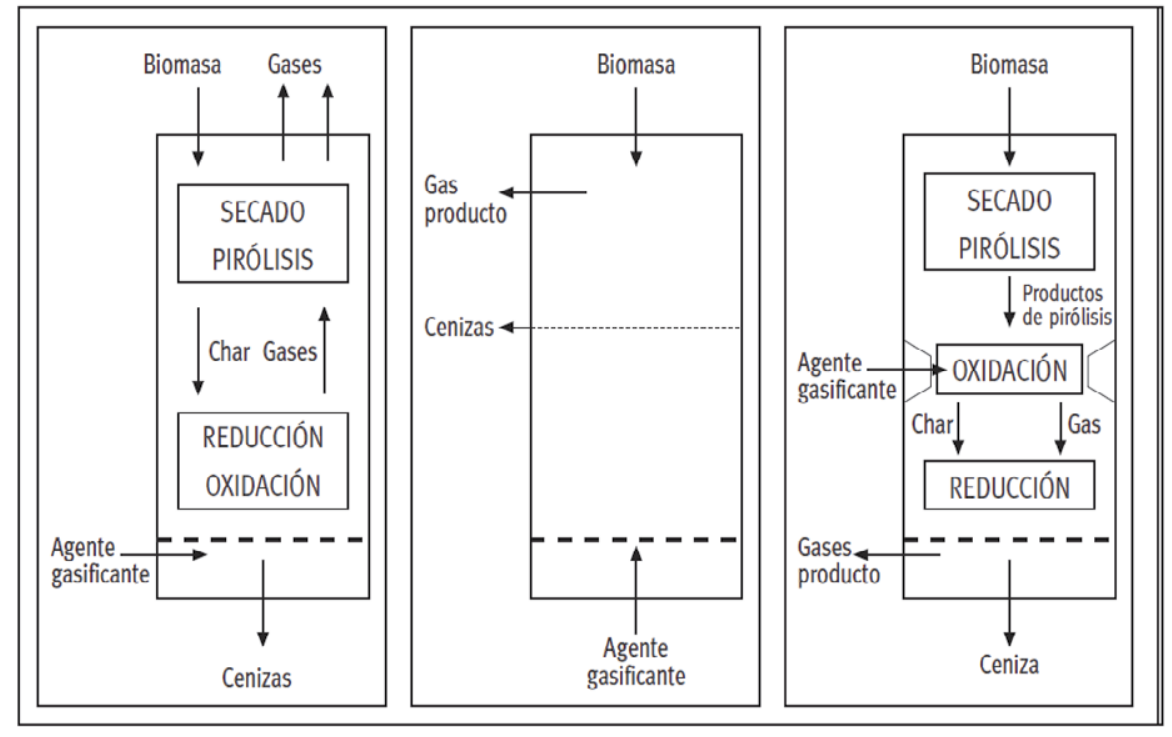

Figura 2. De izquierda a derecha, esquemas de funcionamiento de gasificadores updraft, lecho fluidizado y downdraft

Fuente: (IDEA, 2007).

sólida de biomasa es mantenida en suspensión mediante el gas, generalmente no se puede diferenciar las zonas del proceso; la principal ventaja de este tipo de gasificador es que cada partícula de biomasa, al estar en suspensión, es sometida al proceso en cualquier punto del gasificador, lo cual genera una elevada velocidad de reacción y un perfil de temperatura uniforme que a la larga permite un mejor control en la operación. También posee alta capacidad en términos de tonelada de biomasa por unidad volumétrica del reactor, a diferencia de otros gasificadores, y esto se debe principalmente a la operación bajo alta presión, que permite disminuir el tamaño del gasificador.

En los gasificadores por lecho móvil en contracorriente (updraft), la biomasa entrante y el gas de síntesis se mueven en sentidos contrarios, siendo la biomasa de manera descendente y el gas caliente en forma ascendente; conforme desciende la partícula de biomasa, esta es calentada por la corriente caliente ascendente de gas, hasta llegar a la zona de combustión (oxidación parcial) donde se inicia el proceso de gasificación, la velocidad de descenso de la biomasa se verá condicionada por la tasa de extracción de cenizas, las cuales son 
el subproducto de la gasificación de la biomasa (Elias, 2010).

En los gasificadores de lecho móvil equicorriente (downdraft) el sólido ingresa por la parte superior experimentando procesos de secado y pirólisis al aumentar la temperatura cuando va descendiendo; en la zona inferior se realiza la oxidación mediante combustión parcial teniendo como productos el gas de síntesis, alquitranes y sólidos (char). Parte del gas de síntesis es utilizado para mantener el proceso en operación en la zona de reducción, que se ubica en la parte inferior del reactor, lugar por donde se evacúa el syngas final (Elias, 2010).

Las tecnologías de lecho fluidizado se presenta con mayores ventajas que las de lecho móvil, teniendo como mayores ventajas las características técnicas, escalado, rango de carga, condiciones de arranque y parada, espacio requerido, menor emisión de alquitranes y requerimientos de la biomasa, siendo su principal debilidad la elevada emisión de material particulado, polvo y gran consumo de energía (Elias, 2010).

\section{La generación de energía eléctrica distribuida}

Purchala, et al. (2005), sostienen que actualmente no existe un consenso sobre la definición exacta en lo que respecta a generación distribuida; algunos países la definen según la tensión eléctrica que posee, otros en el principio de que la generación distribuida es cercana al destinatario final, y otros con base en ciertas características propias como el uso de energías renovables, la potencia, la distribución, etc. Ruiz y Ramos (2009) señalan que la generación distribuida se basa en la existencia de un conjunto de instalaciones generadoras de electricidad y calor situadas cerca del usuario final y que pueden funcionar de manera autónoma o alimentar una red de distribución.

Asimismo, Ruiz y Ramos (2009) muestran que el modelo tradicional de generación de energía eléctrica se basa en el paradigma de grandes centrales energéticas alejadas de la zona de los núcleos de consumo y transportadas por una gran red de alta tensión, lo cual genera pérdidas de energía al momento de la transmisión. González-Longatt (2008) indica que otra principal característica de la generación distribuida es el cambio de flujo de energía, dado que en el sistema tradicional el flujo es unidireccional; debido a que la generación distribuida es una forma alternativa de generación, los flujos de generación energética convergerán en el usuario final, lo cual se aprecia en la figura 3. Este tipo de flujo otorga mayores beneficios al consumidor en cuanto a dotación eléctrica, confiabilidad y autonomía.

Por otro lado, a diferencia del sistema tradicional, la configuración de la distribución de la generación distribuida se basa en el modelo de redes circulares provenientes de uno o varios núcleos generadores; esta conformación confiere ciertas ventajas, principalmente de independencia y contingencia ante el daño de un punto de distribución, los cuales pueden ser fácilmente identificados y aislados sin repercusiones en la dotación eléctrica (Ackermann, et al., 2000).

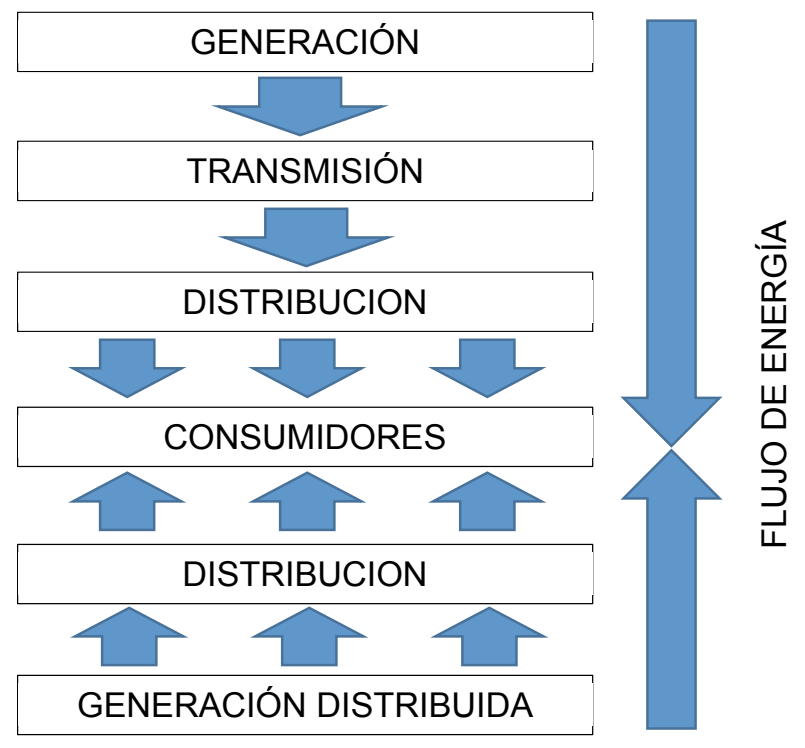

Figura 3. Esquema de flujo de energía mediante el esquema de generación distribuida

Fuente: (González-Longatt, 2008) 
Las principales ventajas de la generación distribuida es que aparece como una opción viable para generar energía eléctrica utilizando recursos renovables de forma eficiente, confiable y de calidad (Carvajal y Marín, 2012) y puede reducir las pérdidas activas y reactivas, mejorar el perfil de tensiones y aliviar la congestión en el sistema eléctrico (Buitrago y López, 2013).

\section{METODOLOGÍA}

El desarrollo metodológico de la presente investigación de realizó en cuatro fases, el cual se presenta de manera resumida en la figura 4 .

\section{Fase 1. Análisis de la cobertura eléctrica en Madre de Dios y determinación de centros poblados sin electrificación rural}

Para la determinación de la cobertura se utilizó la aplicación informática del portal web del Ministerio de Energía y Minas de la República del Perú (MEM), la cual permite visualizar la distribución de las redes eléctricas junto con la tensión correspondiente; a su vez, se puede visualizar los centros poblados que cuentan con proyectos de electrificación rural y caso inverso, tal como se aprecia en la figura 5 .

\begin{tabular}{|c|c|}
\hline $\begin{array}{l}\text { Fase 1: Análisis de la cobertura eléctrica en } \\
\text { Madre de Dios y determinación de centros } \\
\text { poblados sin electrificación rural }\end{array}$ & $\begin{array}{l}\text { Herramienta: Aplicación informática del portal } \\
\text { web del MEM }\end{array}$ \\
\hline $\begin{array}{l}\text { Fase 2: Determinación y cuantificación de la } \\
\text { biomasa residual agrícola y su localización } \\
\text { geográfica }\end{array}$ & $\begin{array}{l}\text { Herramientas: Metodología propuesta por } \\
\text { Velásquez (2006), series históricas de } \\
\text { producción de cultivos (MINAG, 2000-2010) y } \\
\text { IV Censo Agropecuario Nacional - Cenagro } \\
\text { (INEI, 2012) }\end{array}$ \\
\hline $\begin{array}{l}\text { Fase 3: Análisis de las opciones tecnológicas } \\
\text { para el aprovechamiento de la biomasa } \\
\text { residual agrícola. }\end{array}$ & $\begin{array}{l}\text { Herramienta: Análisis de ventajas y } \\
\text { desventajas de opciones tecnológicas de pre- } \\
\text { tratamiento, tratamiento y aprovechamiento } \\
\text { energético }\end{array}$ \\
\hline $\begin{array}{l}\text { Fase 4: Estimación de la cantidad de energía } \\
\text { contenida en la biomasa residual agrícola }\end{array}$ & $\begin{array}{l}\text { Herramientas: Cálculo del poder calorífico } \\
\text { (Nogués et al., 2010), cálculo de la energía } \\
\text { potencial mediante PCl inferior, relación de } \\
\text { cantidad de biomasa/energía eléctrica } \\
\text { producida (De Val, 2007; García-Bacaicoa, et } \\
\text { al., 2002) }\end{array}$ \\
\hline
\end{tabular}

Figura 4. Esquema metodológico ejecutado

Fuente: elaboración propia 


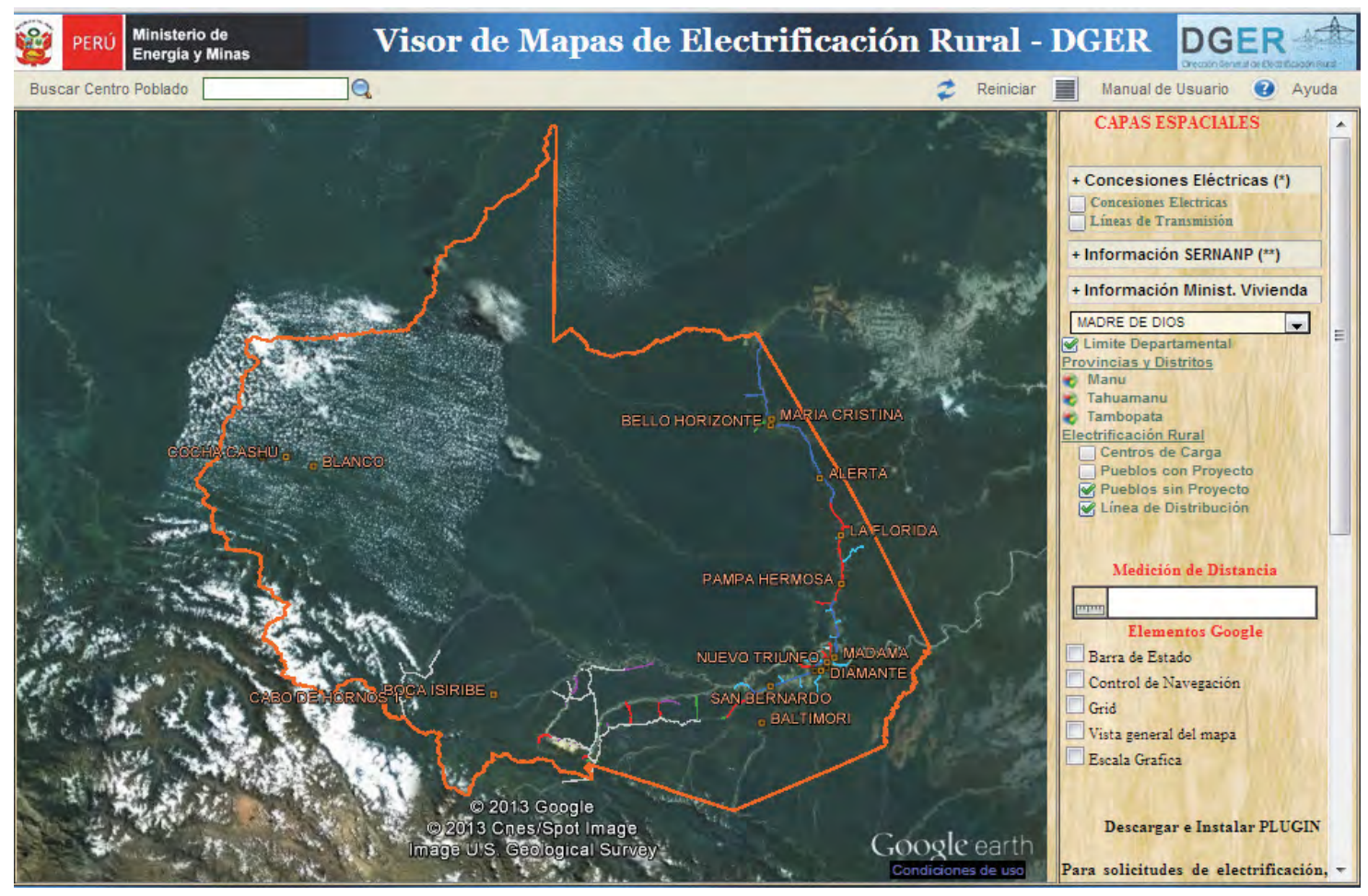

Figura 5. Distribución de redes eléctricas en Madre de Dios

Fuente: (MEM, 2012)

La priorización de los centros poblados se realizó teniendo en consideración las siguientes condicionantes:

- Dispersión geográfica y lejanía a vías comunicación. Existen centros poblados que se encuentran muy dispersos unos de otros, con lo cual no justificaría la conformación de polos de generación de energía distribuida.

- Cantidad de población. La escasa población no es prioritaria para el establecimiento de núcleos de generación, ya que se debe optar siempre por el mayor balance de costo-beneficio.

- Zonas generadoras de biomasa. La generación distribuida se basa en la generación eléctrica in situ, por tanto, no puede considerarse centros poblados cuyas zonas tengan generación mínima o nula de biomasa residual agrícola, que es el insumo primordial para la generación.

\section{Fase 2. Determinación y cuantificación de la biomasa residual agrícola y su localización geográfica}

Para el cálculo de la biomasa residual agrícola, se tomó la metodología propuesta por Velásquez (2006) haciendo uso de las series históricas del MINAG entre 2000-2010, por ser estos de la década más reciente.

Una vez identificado los principales cultivos, se procedió a encontrar los factores de conversión entre la producción total y el tipo y cantidad de residuo generados. Seguidamente, haciendo uso de los datos de producción agrícola a nivel distrital especificados por el IV Censo Nacional Agropecuario-Cenagro (INEI, 2012), se estimó la cantidad de biomasa residual agrícola generada para los cultivos principales a nivel de distritos por correspondencia proporcional. Por último, se integró la información 
obtenida con información geográfica de los frentes económicos (IIAP, 2001), a través del software ArcGIS, y así se determinó las zonas generadoras de biomasa residual agrícola en los distritos con conforman el departamento de Madre de Dios.

\section{Fase 3. Análisis de las opciones tecnológicas para el aprovechamiento de la biomasa residual agrícola}

Se analizó las opciones tecnológicas disponibles para pretratamiento, tratamiento y aprovechamiento energético; este análisis consistió en la revisión de ventajas y desventajas, madurez tecnológica, eficiencia, facilidad de implementación y mantenimiento de los distintos tipos de tecnología que se encuentran en el mercado, considerando también las condiciones logísticas y climáticas de la zona de estudio.

\section{Fase 4. Estimación de la cantidad de energía contenida en la biomasa residual agrícola}

La estimación de la cantidad de energía contenida en la biomasa residual agrícola siguió la siguiente secuencia.

\section{Cálculo del poder calorífico}

Se determinó el poder calorífico de la biomasa residual agrícola considerada en sus dos expresiones: poder calorífico superior (PCS) y poder calorífico inferior ( $\mathrm{PCl})$. A su vez, cada una de estas expresiones fue estimada tanto en base húmeda como en base seca, siguiendo las fórmulas señaladas por Nogués, et al. (2010), quien cita a su vez a Milne (1978). El PCS en base seca se halló mediante la ecuación (3).

$$
P C S_{b s}\left(\frac{M J}{k g}\right)=0.314 \times C+1.322 \times H-0.12 \times O-0.12 \times N+0.0686 \times S-0.0153 \times Z
$$

Donde $\mathrm{C}, \mathrm{H}, \mathrm{O}, \mathrm{N}$ y $\mathrm{S}$ son las fracciones másicas en base seca, de carbono, hidrógeno, oxígeno, nitrógeno y azufre respectivamente en el combustible, y Z la fracción de las cenizas en base seca.

El PCI se calculó a partir del PCS mediante la ecuación (4).

$$
P C I_{b s}=P C S_{b s}-\left(9 \times 2.449 \times H_{b s}\right)
$$

Donde $\mathrm{H}_{\mathrm{bs}}$ es la fracción másica del hidrógeno en el combustible en base seca y los poderes caloríficos están expresados como kJ/kg.

Fue necesario, también, considerar los valores del PCS y PCl en base húmeda, ya que el contenido de humedad va a reducir la energía disponible. Los valores del PCS y PCI en base húmeda y seca están correlacionados mediante las ecuaciones (5) y (6).

$$
\mathrm{PCI}_{\mathrm{bh}}=\mathrm{PCS}_{\mathrm{bs}} \times\left(1-\frac{\mathrm{W}_{\mathrm{bh}}}{100}\right)
$$

$$
\mathrm{PCI}_{\mathrm{bh}}=\mathrm{PCS}_{\mathrm{bs}}-2.449 \times\left[\mathrm{W}_{\mathrm{bh}}+9 \times \mathrm{H}_{\mathrm{bs}} \times\left(1-\frac{\mathrm{W}_{\mathrm{bh}}}{100}\right)\right]
$$

$W_{b h}$ el porcentaje de humedad en base húmeda, $H_{b s}$ la fracción másica en base seca el combustible y los poderes caloríficos expresados en $\mathrm{KJ} / \mathrm{kg}$.

\section{Cálculo de la energía potencial}

Una vez hallado el $\mathrm{PCl}$ de la biomasa residual agrícola, la determinación de la energía potencial química no es más que la multiplicación del $\mathrm{PCI}$ por la cantidad de biomasa generada. Se escogió el $\mathrm{PCl}$ en base húmeda debido a que esta expresión es más acorde a la realidad.

\section{Relación de producción energética: cantidad} de biomasa/energía eléctrica producida Para determinación de energía aprovechable para la producción eléctrica distribuida fue necesario determinar la relación entre la cantidad de biomasa y la energía neta que se puede obtener de la misma; para ello, se realizaron estimaciones de las 
tecnologías gasificación por de lecho móvil y fluidizado, tomando en consideración los resultados de García-Bacaicoa, et al. (2002), y Del Val (2007).

Para determinar esta relación se consideró la turbina a gas como tecnología de aprovechamiento energético, considerando un 35\% de eficiencia energética sobre el $\mathrm{PCl}$ de syngas producido.

\section{RESULTADOS}

La determinación de la cobertura de electrificación rural en Madre de Dios por medio de la aplicación informática de la web del MEM arrojó un resultado de diecinueve centros poblados sin electrificación rural; de ellos se priorizaron cinco centros poblados, teniendo en cuenta el mayor número de viviendas, proximidad con la carretera interoceánica y generación de biomasa local. Los centros poblados priorizados se encuentran detallados en la tabla 1.

Analizando las series históricas oficiales del MINAG, se obtuvo que los principales cultivos en la región Madre de Dios durante 2000 y 2010 fueron el plátano, el maíz amarillo duro y el arroz, y en menor cantidad los cultivos de achiote, café, coco, limón, naranja, palta, papaya, piña, soya y tomate, tal como se especifica en la tabla 2. Para esta investigación solo se consideraron los tres mayores de los antes mencionados, tal como se observa en la figura 6 .

Tabla 1. Inventario de centros poblados rurales prioritarios de Madre de Dios sin proyecto de electrificación rural

\begin{tabular}{lccccc}
\hline $\begin{array}{l}\text { Nombre del centro } \\
\text { poblado }\end{array}$ & $\begin{array}{c}\text { Número de viviendas } \\
\text { (INEI, 2007) }\end{array}$ & \multicolumn{2}{c}{ Coordenadas utm, wgs-84 zona 19 } & Zona & \multirow{2}{*}{$\begin{array}{c}\text { Altitud } \\
\text { (m.s.n.m.) }\end{array}$} \\
\cline { 5 - 6 } & 41 & Este & Norte & & \\
\hline Bello Horizonte & 21 & 445785 & 8739960 & $19 \mathrm{~L}$ & 308 \\
\hline María Cristina & 21 & 446355 & 8743432 & $19 \mathrm{~L}$ & 304 \\
\hline Nuevo Triunfo & 17 & 480388 & 8609088 & $19 \mathrm{~L}$ & 193 \\
\hline Nuevo Sol Naciente & 30 & 480209 & 8605648 & $19 \mathrm{~L}$ & 191 \\
\hline Diamante & 477043 & 8604263 & $19 \mathrm{~L}$ & 203 \\
\hline
\end{tabular}

Fuente: Adaptado de (MEM, 2013)

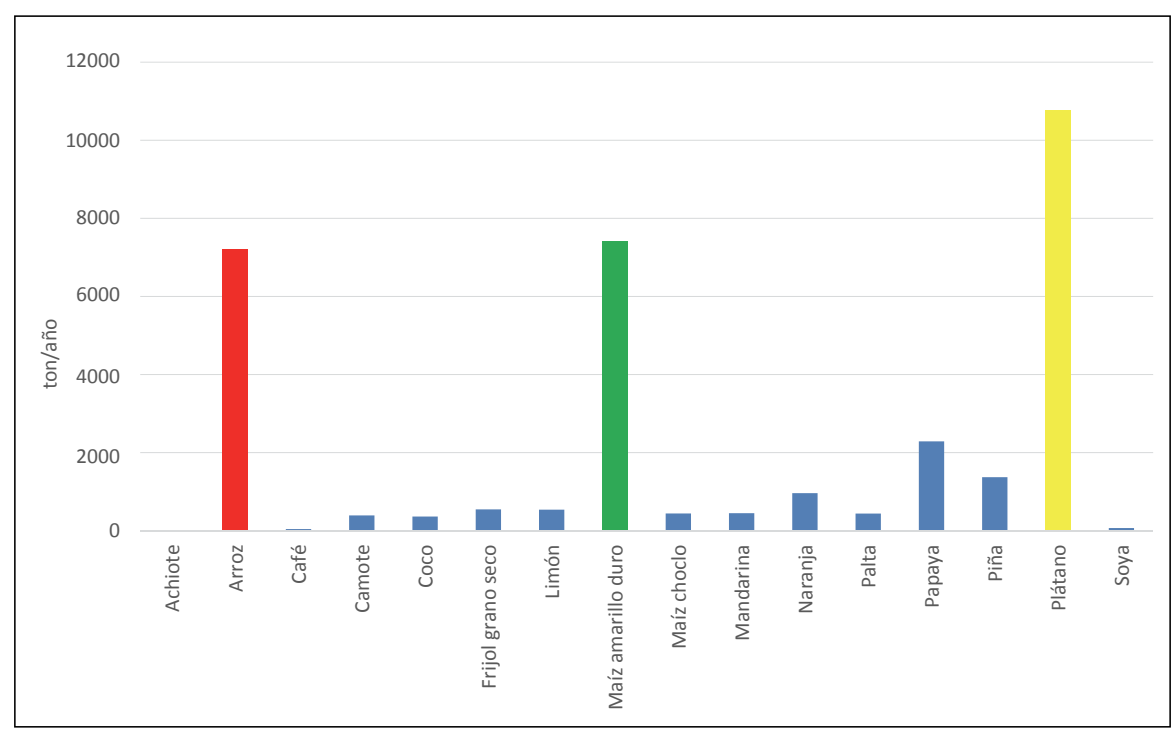

Figura 6. Principales cultivos en la región Madre de Dios, promedio del 2000-2010

Fuente: elaboración propia 
Tabla 2. Producción agrícola de Madre de Dios, valores promedio 2000-2010 (t/año)

\begin{tabular}{lccc}
\hline \multicolumn{1}{c}{ Cultivo } & Producción promedio (t/año) & Cultivo & Producción promedio (t/año) \\
\hline Achiote & 7.45 & Maíz choclo & 371.09 \\
\hline Arroz & $7,222.91$ & Mandarina & 376.82 \\
\hline Café & 34.18 & Naranja & 810.36 \\
\hline Camote & 329.18 & Palta & 368.55 \\
\hline Coco & 304.82 & Papaya & $1,930.55$ \\
\hline Frijol grano seco & 459 & Piña & $1,155.73$ \\
\hline Limón & 452.91 & Plátano & $10,770.36$ \\
\hline Maíz amarillo duro & $7,416.25$ & Soya & 55.63 \\
\hline
\end{tabular}

Fuente: (MINAG, 2013)

Una vez identificado los principales cultivos, se procedió a encontrar los factores de conversión entre la producción total y el tipo y cantidad de residuo generado, que fueron hallados en fuentes de diversos autores y centros de investigación como se muestra en la tabla 3, tabla 4 y tabla 5 .

Tal como se mencionó en la metodología, se encontró el valor promedio de los factores de

Tabla 3. Factores de residuos para el cultivo de arroz

\begin{tabular}{cccc}
\hline Cultivo & Tipo de residuo & Factor & Fuente \\
\hline \multirow{4}{*}{ Arroz } & Paja & 0.7 & CIRCE, 2006. \\
\cline { 2 - 4 } & Paja & 0.7 & CORPOEMA, 2010. \\
\cline { 2 - 4 } & Cascarilla & 0.29 & Rincón, 2009. \\
\cline { 2 - 4 } & Cascarilla & 0.17 & CORPOEMA, 2010. \\
\cline { 2 - 4 } & Cascarilla & 0.22 & García, et al, 2010. \\
\hline
\end{tabular}

Fuente: elaboración propia

Tabla 4. Factores de residuos para el maíz amarillo duro

\begin{tabular}{llll}
\hline Cultivo & Tipo de residuo & Factor & \multicolumn{1}{c}{ Fuente } \\
\hline \multirow{2}{*}{$\begin{array}{l}\text { Maíz } \\
\text { amarillo } \\
\text { duro }\end{array}$} & Cañote & 0.65 & CIRCE, 2006. \\
\cline { 2 - 4 } & Coronta & 0.21 & CORPOEMA, 2010. \\
\cline { 2 - 4 } & Hojas y tallos & 0.48 & CORPOEMA, 2010. \\
\cline { 2 - 4 } & Hojas tallos & 0.5 & Manterola, et al,1999. \\
\hline
\end{tabular}

Fuente: elaboración propia

Tabla 5. Factores de residuos para el plátano

\begin{tabular}{cccc}
\hline Cultivo & Tipo de residuo & Factor & Fuente \\
\hline \multirow{2}{*}{ Plátano } & Vástago & 0.71 & CORPOEMA, 2010. \\
\cline { 2 - 4 } & Vástago & 0.75 & Pérez, 1994. \\
\hline
\end{tabular}

Fuente: elaboración propia 
generación de residuos de los cultivos principales, los cuales se detallan en la tabla 6

Tabla 6. Factores de residuos promediados

\begin{tabular}{lcc}
\hline \multicolumn{1}{c}{ Cultivo } & Tipo De Residuo & Factor* \\
\hline \multirow{2}{*}{ Arroz } & Paja & 0.7 \\
\cline { 2 - 3 } & Cascarilla & 0.22 \\
\hline \multirow{2}{*}{ Maíz amarillo duro } & Coronta & 0.2 \\
\cline { 2 - 3 } & Hojas y tallos & 0.5 \\
\hline Plátano & Vástago & 0.73 \\
\hline
\end{tabular}

*Factor dado en tonelada de residuo/tonelada de producto. Se considera "producto" al cultivo ya cosechado que incluye tanto la parte apreciable como los residuos, de tal manera que el factor debe ser menor que la unidad.

Fuente: elaboración propia
Con los factores de generación de biomasa ya establecidos, se realizó el cálculo de la generación potencial promedio de biomasa residual agrícola, a nivel regional, cuyos resultados promedio se presentan en la tabla 7.

Posteriormente, se realizó la estimación a nivel distrital haciendo uso de los datos del IV Censo Nacional Agropecuario (Cenagro; INEI, 2012), cuyos resultados se detallan en la tabla 8 .

Complementariamente, se determinó las zonas geográficas que generan biomasa residual agrícola con base a la información procedente del Instituto de Investigación de la Amazonía Peruana (IIAP, 2001), la cuales son especificadas en la figura 7.

Tabla 7. Biomasa potencial promedio generada a nivel de región

\begin{tabular}{lll}
\hline \multicolumn{1}{c}{ Cultivo } & \multicolumn{1}{c}{$\begin{array}{c}\text { Producción anual promedio } \\
\text { (ton/año) }\end{array}$} & $\begin{array}{c}\text { Biomasa generada promedio } \\
\text { (ton/año) }\end{array}$ \\
\hline Arroz & $7,222.91$ & $6,645.08$ \\
\hline Maíz amarillo duro & $7,416.00$ & $5,191.20$ \\
\hline Plátano & $10,770.36$ & $7,862.37$ \\
\hline
\end{tabular}

Fuente: elaboración propia

Tabla 8. Biomasa potencial promedio generada a nivel de distritos

\begin{tabular}{|c|c|c|c|c|}
\hline \multirow{2}{*}{ Distrito } & \multirow{2}{*}{ 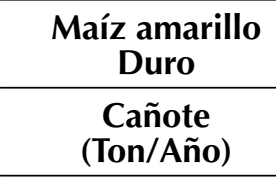 } & \multicolumn{2}{|c|}{ Arroz } & \multirow{2}{*}{$\begin{array}{c}\text { Plátano } \\
\begin{array}{c}\text { Vástago } \\
\text { (Ton/Año) }\end{array} \\
\end{array}$} \\
\hline & & $\begin{array}{c}\text { Paja } \\
\text { (Ton/Año) }\end{array}$ & $\begin{array}{l}\text { Cascarilla } \\
\text { (Ton/Año) }\end{array}$ & \\
\hline Tambopata & 691,34 & 658,61 & 206,99 & N.R. \\
\hline Inambari & 517,14 & 737,45 & 231,77 & N.R. \\
\hline Las piedras & 940,52 & 981,12 & 308,35 & N.R. \\
\hline Laberinto & 329,31 & 594,39 & 186,81 & N.R. \\
\hline Manu & 163,22 & 51,29 & 16,12 & N.R. \\
\hline Fitzcarrald & 64,93 & 9,20 & 2,89 & N.R. \\
\hline Madre de Dios & 102,04 & 77,73 & 24,43 & N.R. \\
\hline Huepetuhe & 58,67 & N.R. & N.R. & N.R. \\
\hline Iñapari & 432,17 & 185,38 & 58,26 & N.R. \\
\hline Iberia & 1270,44 & 968,54 & 304,40 & N.R. \\
\hline Tahuamanu & 621,42 & 792,34 & 249,02 & N.R. \\
\hline
\end{tabular}

Fuente: elaboración propia

*N.R.: No registrado en el IV Censo Nacional Agropecuario (Cenagro) 2012 


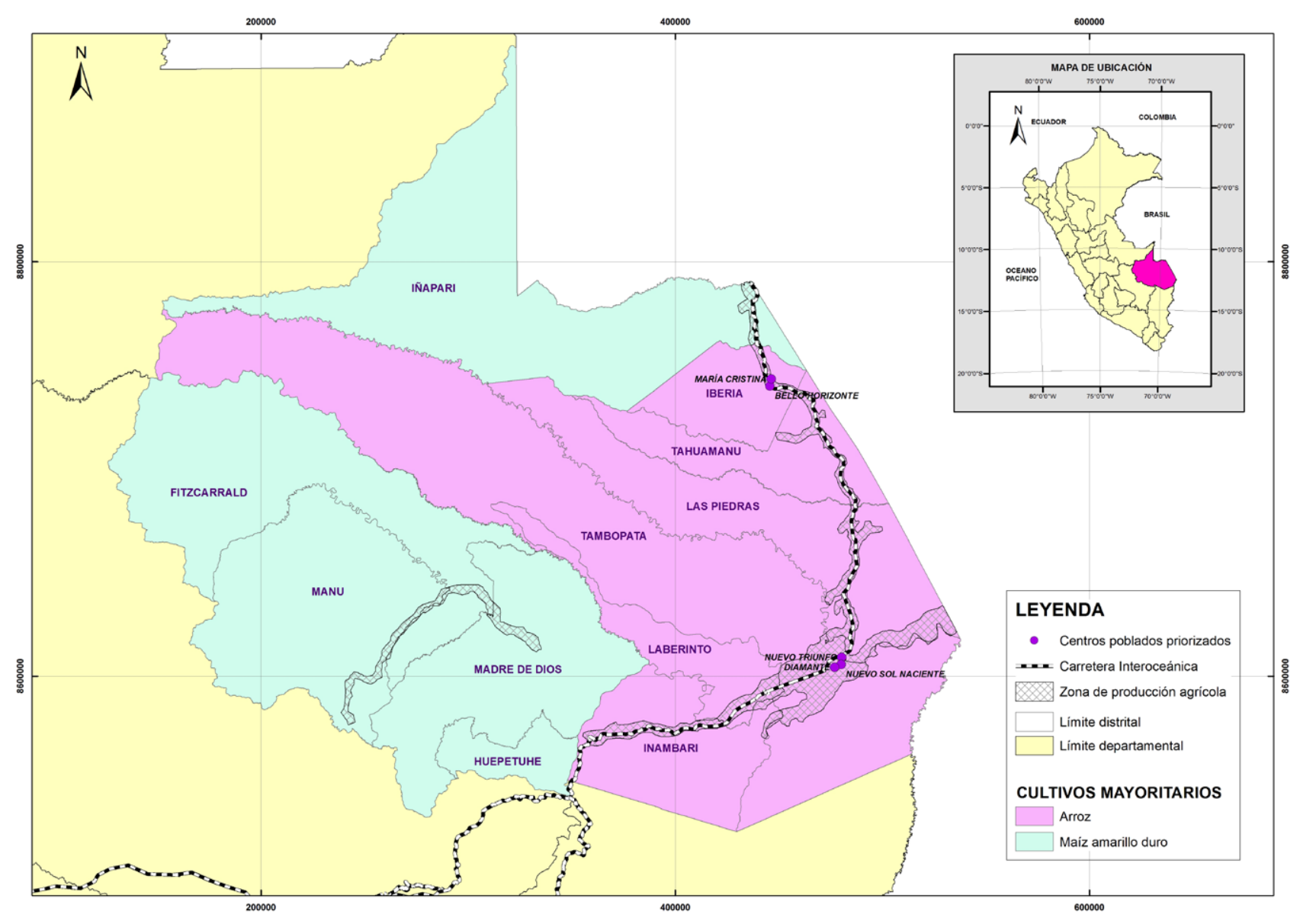

Figura 7. Zonas agrícolas generadores de biomasa residual agrícola en Madre de Dios

Fuente: elaboración propia

A partir de los datos de composición elemental y de contenido de humedad se realizó la estimación del $\mathrm{PCl}$ y PCS, tanto en base húmeda como seca, cuyos resultados se muestran en la tabla 9.

Teniendo en cuenta el $\mathrm{PCl}$ en base húmeda, dado que este es el valor más acorde a la realidad, se calculó la energía potencial promedio de la biomasa residual agrícola considerada la cual se muestra en la tabla 10.

Esta energía potencial no puede aprovecharse al $100 \%$ dado que las tecnologías poseen un porcentaje de conversión. Adicionalmente, los tipos de biomasa estudiada deben poseer ciertos criterios técnicos para ser considerados como aprovechables. Nogués, et al. (2010), cita tres criterios a evaluar para tal fin.

Disponibilidad logística: Según la FAO (2010), la paja de arroz, la coronta, hojas y tallos de maíz suelen ser utilizados como forraje para el ganado. En cuanto a los vástagos de plátano son desechos en campo y hay evidencia de que suele ser utilizado como materia prima para elaboración de sogas. No se ha encontrado evidencia de uso agropecuario de cascarilla de arroz por lo cual se consideró este tipo de residuos como el más apropiado para su aprovechamiento.

Humedad: Su consideración es importante para la generación de energía ya que mayor cantidad 
Tabla 9. PCS y PCl en base húmeda y seca de los residuos a estudiar

\begin{tabular}{llllll}
\hline \multirow{2}{*}{ Cultivo } & \multirow{2}{*}{$\begin{array}{c}\text { Tipo de } \\
\text { residuo }\end{array}$} & \multicolumn{2}{c}{ Base húmeda } & \multicolumn{2}{c}{ Base seca } \\
\cline { 3 - 6 } & Cascarilla & 12.76 & 12.63 & 14.26 & 14.15 \\
\hline Arroz & Pag) & PCI $(\mathbf{M J} / \mathbf{k g})$ & PCS $(\mathbf{M J} / \mathbf{k g})$ & $\mathbf{P C I}(\mathbf{M J})$ \\
\hline Arroz & Paja & 12.93 & 12.79 & 14.59 & 14.47 \\
\hline Maíz & Cañote & 14.10 & 13.96 & 16.41 & 16.28 \\
\hline Plátano & Vástago & 1.00 & 0.77 & 15.69 & 15.56 \\
\hline
\end{tabular}

Fuente: elaboración propia

Tabla 10. Energía potencial de la biomasa residual agrícola considerada

\begin{tabular}{llll}
\hline \multicolumn{4}{c}{ Energía potencial promedio $\mathbf{( M W h / a n ̃ o ) ~}$} \\
\hline Cascarilla de arroz & Paja de arroz & Vástago de plátano & Cañote de maíz \\
\hline $5,619.48$ & $18,106.68$ & $1,695.13$ & $20,291.36$ \\
\hline
\end{tabular}

Fuente: elaboración propia

de humedad reduce el poder calorífico de la biomasa. Según Nogués, et al. (2010), el contenido de humedad adecuado para aprovechar la biomasa residual agrícola no debe sobrepasar el 30\%, siendo contenido óptimo menor al 15\%, valor que es cumplido por la cascarilla de arroz, cuyo porcentaje de humedad es del 10,55 \% (Valverde, et al., 2007).

Exportación de cultivos: Según Fernández (2010), la producción agrícola en Madre de Dios se destina principalmente al autoconsumo y el pequeño excedente al comercio local. Esta región es deficitaria en producción agrícola, por lo que debe abastecerse de productos de la sierra y la costa. Por tanto, se puede afirmar que la biomasa generada en Madre de Dios permanece inamovible dentro de los límites de la región.

Teniendo en consideración estos tres criterios, se determinó que el residuo de categoría aprovechable más apropiado para generación de energía es la cascarilla de arroz, ya que no tiene uso agropecuario posterior y posee la cantidad adecuada de humedad; sin embargo, existe la posibilidad de aprovechamiento de los demás tipos de biomasa mencionados siempre y cuando se pueda aplicar la logística adecuada para su recolección y adecuación.

Para la estimación de la energía aprovechable de la cascarilla de arroz se consideró la gasificación como opción tecnológica en los dos tipos de familia que involucra: lecho móvil y lecho fluidizado. Adicionalmente, se consideró el sacador solar como pretratamiento óptimo de la cascarilla de arroz y turbina a gas como tecnología de mayor aprovechamiento energético.

Para la cuantificación de la energía aprovechable se utilizó dos parámetros de rendimiento: MCE para gasificadores de lecho móvil (De Val, 2007) y Rto a gas para lecho fluidizado (García-Bacaicoa, et al., 2002). El parámetro MCE mide la relación entre la masa del syngas producido por la biomasa alimentada y masa de aire alimentado, con lo cual se pudo determinar la relación entre la energía aprovechable y la biomasa alimentada. El parámetro Rto a gas mide el volumen de syngas producido por kilogramo de biomasa seca alimentada, con el cual se pudo determinar el rango de generación de energía aprovechable por unidad de biomasa alimentada. La tabla 11 muestra el resultado de las estimaciones para ambas familias de gasificadores. 
Haciendo uso del rango obtenido en la tabla 11 y los datos de la producción de biomasa residual agrícola por distritos detallada en la tabla 8, se estimó la energía aprovechable a nivel distrital en la región Madre de Dios, cuyo resultado se detalla en la tabla 12.

Dado que la presente investigación apunta al establecimiento de polos de generación de energía, es necesario priorizar los distritos cuyas zonas generadoras se encuentren próximas a los centros poblados priorizados. En este sentido, revisando la figura 7 , se aprecia que las zonas generadoras de cascarilla de arroz de los distritos Fitzcarrald, Manu, Madre de Dios y Huepetuhe se encuentran lejanas a la carretera interoceánica y a los centros poblados priorizados, además de tener una producción de arroz menor, por lo cual se considera conveniente descartarlas para el aprovechamiento energético. De esta forma, la energía total aprovechable se situaría entre 1,296.54 a 2,029.00 MWh/año por medio de lecho móvil, y entre 491.49 a 1,387.26 MWh/año por medio de lecho fluidizado.

Según los datos del MEM (2010), la región de Madre de Dios posee un consumo eléctrico per cápita de 326.1 kWh promedio en el año 2010. Considerando el número de viviendas en la tabla 1 y asumiendo que el tamaño de una vivienda es de cinco personas por promedio, que significa 650 habitantes en 130 viviendas, el consumo anual asciende a 211.9 MWh al año. Teniendo en cuenta que la energía aprovechable se encuentra por encima de 491.49 MWh/año, se puede concluir que la demanda energética de los centros poblados de María Cristina, Bello Horizonte, Nuevo Sol Naciente, Nuevo Triunfo y Diamante se podría

Tabla 11. Comparación de energía aprovechable por lecho móvil y lecho fluidizado para la cascarilla de arroz en la región Madre de Dios

\begin{tabular}{lcc}
\hline & \multicolumn{2}{c}{ Energía aprovechable (KWh/ton) } \\
\hline & Lecho móvil & Lecho fluidizado \\
\hline Rango & $838.86-1,319.23$ & $317.99-897.56$ \\
\hline Valor medio & $1,079.38$ & 629.99 \\
\hline
\end{tabular}

Fuente: elaboración propia

Tabla 12. Energía aprovechable de cascarilla de arroz mediante lecho móvil y lecho fluidizado según los distritos de Madre de Dios

\begin{tabular}{lcc}
\hline \multirow{2}{*}{ Distrito } & \multicolumn{2}{c}{ Energía (MWh/año) } \\
\cline { 2 - 3 } & Lecho móvil & Lecho fluidizado \\
\hline Tambopata & $173.64-273.07$ & $65.82-185.79$ \\
\hline Inambari & $194.42-305.76$ & $73.7-208.03$ \\
\hline Las Piedras & $258.66-406.79$ & $98.05-276.76$ \\
\hline Laberinto & $156.71-246.44$ & $59.4-167.67$ \\
\hline Manu & $13.52-21.26$ & $5.13-14.47$ \\
\hline Fitzcarrald & $2.42-3.81$ & $0.92-2.59$ \\
\hline Madre de Dios & $20.49-32.23$ & N.R. \\
\hline Huepetuhe & N.R. & $18.53-52.29$ \\
\hline Iñapari & $48.87-76.86$ & $96.8-273.21$ \\
\hline Iberia & $255.35-401.57$ & $79.19-223.51$ \\
\hline Tahuamanu & $208.89-328.51$ & $505.31-1426.25$ \\
\hline Total & $1332.97-2096.3$ &
\end{tabular}

Fuente: elaboración propia 
cubrir al 100\%, considerando el aporte de biomasa residual de cascarilla de arroz de los distritos de Inambari, Tambopata, Las Piedras, Laberinto, Tahuamanu, Iberia e Iñapari.

Sobre la base de los resultados, se propone el establecimiento de dos polos de generación distribuida: uno en la zona norte denominado "Iberia", ubicado en el distrito del mismo nombre, conformado por los centros poblados de Bello Horizonte y María Cristina, y otro en la zona sur denominado "Puerto Maldonado", ubicado en el distrito de Tambopata, conformado por los centros poblados de Nuevo Triunfo, Nuevo Sol Naciente y Diamante (figura 8).

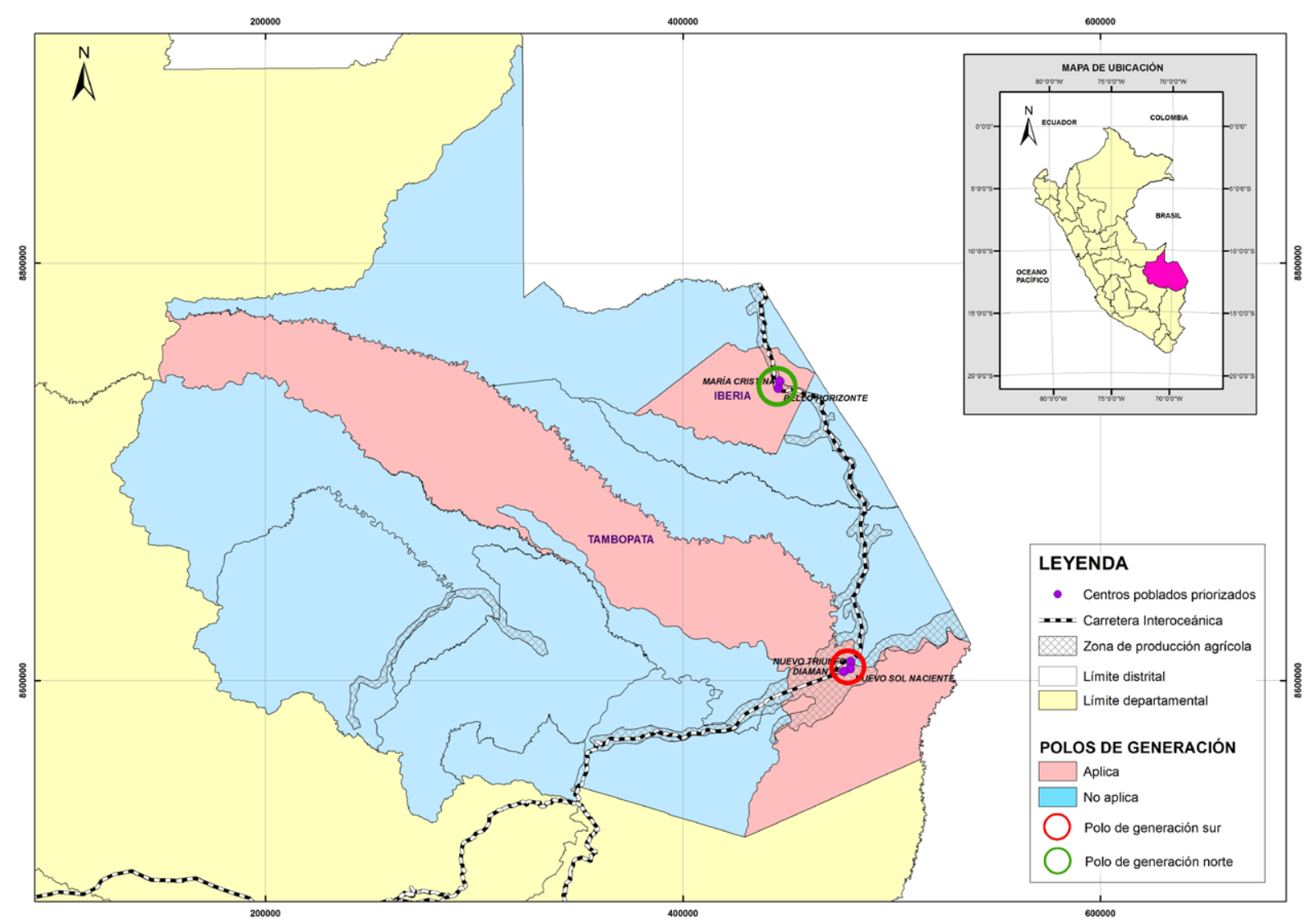

Figura 8. Polos de generación propuestos en Madre de Dios

Fuente: elaboración propia

\section{CONCLUSIONES}

La región de Madre de Dios cuenta con potencial de generación de energía eléctrica distribuida que puede cubrir la demanda de los cinco centros poblados: María Cristina, Bello Horizonte, Nuevo Sol Naciente, Nuevo Triunfo y Diamante al 100\% a través del aprovechamiento de la cascarilla de arroz proveniente de siete de sus once distritos por medio de la utilización de gasificadores tanto de lecho fluidizado como de lecho móvil.

Los centros poblados María Cristina y Bello Horizonte conformarían un polo de energía eléctrica distribuida ubicado en la zona norte, mientras 
que los centros poblados Sol Naciente, Nuevo Truinfo y Diamante conformarían el polo ubicado en la zona sur.

Asimismo, la energía generada en la región Madre de Dios mediante lecho fluidizado podría atender la demanda de 1495 habitantes aproximadamente, mientras que mediante lecho móvil se cubriría la demanda de 3945 habitantes aproximadamente.

El potencial de la generación de energía aprovechable por tonelada de cascarilla de arroz se encuentra en el rango de 838.86 a 1319.23 KWh mediante gasificadores de lecho móvil, y de 317.99 a $897.56 \mathrm{KWh}$ por lecho fluidizado.

\section{AGRADECIMIENTOS}

Al señor Luis Alonso Salazar Pacheco por su asesoría y apoyo profesional en la elaboración de mapas temáticos mediante el uso del ArcGIS.

\section{REFERENCIAS}

Ackermann, T., Anderson, G., Söder, L. (2000). Distributed Generation: a Definition. Electric Power Systems Research, 57, 195-204.

Buitrago, L. y López, J. (2013). Valoración de los impactos técnicos de la generación distribuida en sistemas de energía eléctrica. Revista Tecnura, 17(36), 50-60.

Casilda, R. (2002). Energía y desarrollo económico en Latinoamérica. Boletín Económico ICE, 2750, 31-44.

Carvajal, S. y Marín, J. (2012). Impacto de la generación distribuida en el sistema eléctrico de potencia colombiano: un enfoque dinámico. Revista Tecnura, 17(35), 77-89.

CIRCE (Centro de Investigación de Recursos y Consumos Energéticos). (2006). Evaluación del potencial de biomasa residual en los ecosistemas forestales y los medios agrícolas en la provincia de Huesca. España: Fundación CIRCE, Universidad de Zaragoza.

CORPOEMA (Corporación para la Energía y el Medio Ambiente). (2010). Formulación de un plan de desarrollo para las fuentes no convencionales de energía en Colombia (PDFNCE). Diagnóstico de las FNCE en Colombia, 2. Colombia: CORPOEMA.

De Juana, J., De Francisco, A., Santos, F., Herrero, M. y Crespo, A. (2007). Energías renovables para el desarrollo. España: Editorial Thompson-Paraninfo.

Del Val, V. (2007). Planta de cogeneración mediante gasificación de biomasa residual. España: Escuela Técnica Superior de Ingenieros Industriales. Universidad de Valladolid,

Elias, X. (2010). Tratamiento y valorización energética de los residuos. España: Editorial Díaz de Santos.

FAO (Organización de las Naciones Unidas para la Agricultura y Alimentación, IT). (2010). Bioenergía y seguridad alimentaria "BEFS": El análisis de BEFS para el Perú-El proyecto BEFS: Apoyo a la política bioenergética del Perú.

Fernández, L. (2010). Desarrollo territorial en Madre de Dios. Los impactos socioambientales de la carretera Interoceánica Sur. Perú: ProNaturaleza-Fundación peruana para la conservación de la naturaleza.

García, A., Contreras, L. y Romero, O. (2010). Incremento de la eficiencia de la empresa arrocera por aprovechamiento energético de residuos. Revista Infociencia, 14(3), 13-24.

García-Bacaicoa, P., Mastral, J., Serrano, S. y Berrueco, C. (2002). Aprovechamiento de biomasa y residuos: actividades del Grupo de Biomasa de la Universidad de Zaragoza. En Congreso de Calidad Ambiental (pp 307-310). España: Ediciones Universidad de Salamanca.

González-Longatt, F. (2008). Generación distribuida: una introducción. Recuperado de http://fglongatt. org/OLD/Archivos/Archivos/SistGD/PPTIntro.pdf

IDAE (Instituto para la Diversificación y Ahorro de Energía, ES). (2007). Biomasa: Gasificación. España: Gobierno de España, Ministerio de Industria, Turismo y Comercio.

IIAP (Instituto de Investigaciones de la Amazonía Peruana, PE). (2001). Madre de Dios: Camino al desarroIlo sostenible-propuesta de zonificación ecológica económica como base para el ordenamiento territorial. Mapa 10: Frentes económicos.

INEI (Instituto Nacional de Estadística e Informática, PE). (2007). XI Censo de Población y VI de vivienda. 
Recuperado de http://desa.inei.gob.pe/censos2007/ tabulados/?id=ResultadosCensales

INEI (Instituto Nacional de Estadística e Informática, PE). (2012). IV Censo Nacional Agropecuario. Recuperado de http://censos.inei.gob.pe/Cenagro/ redatam/

Manterola, H., Cerda, D. y Mira, J. (1999). Los residuos agrícolas y su uso en la alimentación de rumiantes. Chile: Fundación para la Innovación Agraria, Ministerio de Agricultura.

MINAG (Ministerio de Agricultura, PE). (2013). Registros estadísticos. Series históricas. Recuperado de http:// frenteweb.minag.gob.pe/sisca/?mod=consulta_cult

MEM (Ministerio de Energía y Minas, PE). (2013). Portal de electrificación rural. Recuperado de http://intranet2.minem.gob.pe/filegoogle/Electrificacionrural. $\mathrm{html}$

MEM (Ministerio de Energía y Minas, PE). (2010). Estadística eléctrica por regiones (Parte 2). Perú: Autor.

Nogués, F., García-Galindo, D., y Rezeau, A. (2010). Energía de la Biomasa. Volúmen 1. España: Editorial Prensas Universitarias de Zaragoza. Universidad de Zaragoza.

Pérez, M. (1994). Política cubana de recuperación de todo tipo de desperdicios y subproductos para la producción porcina y saneamiento ambiental. Tratamiento y utilización de residuos de origen animal, pesquero y alimenticio en la alimentación animal.
La Habana: Instituto de Investigaciones Porcinas (IPP).

Purchala, K., Belmans, R., Exarchakos, L. y Hawkes, A. (2005). Distributed Generation and The Grid Integration Issues. Recuperado de http://www.eusustel.be/public/documents_publ/WP/WP3/WP\%20 $3.4 .1 \% 20$ Distributed $\% 20$ generation $\% 20$ and $\% 20$ grid\%20integration\%20issues.pdf

Rincón, S. (2009). Generación energética a partir de biomasa residual colombiana. Grupo de Investigación en Biomasa y Optimización Térmica de Procesos (BIOT). Colombia: Universidad Nacional de Colombia.

Ruiz, J. y Ramos, P. (2009). Cambio climático, ¿un desafío a nuestro alcance?. España: Universidad de Salamanca.

Valverde, A., Sarria, B. y Monteagudo, J. (2007). Análisis comparativo de las características fisicoquímicas de la cascarilla de arroz. Scientia et Technia. 13(37), 255-260.

Velásquez, B. (2006). Aprovechamiento de residuos forestales para uso energético. Valencia: Editorial Universidad Politécnica de Valencia.

Velásquez, H., Ruíz, A. y De Oliveira, S. (2010). Análisis energético y exergético del proceso de obtención de etanol a partir de la fruta del banano. Revista Facultad de Ingeniería Universidad de Antioquía, 51, 87-96. 